Pacific

Journal of

Mathematics

THE ESSENTIAL NORMS AND SPECTRA OF COMPOSITION OPERATORS ON $H^{\infty}$

LiXIN ZHENG

Volume $203 \quad$ No. 2

April 2002 


\title{
THE ESSENTIAL NORMS AND SPECTRA OF COMPOSITION OPERATORS ON $H^{\infty}$
}

\author{
LIXIN ZHENG
}

\begin{abstract}
This paper gives a complete characterization of the spectra of composition operators acting on $H^{\infty}$ in the case that the symbol $\varphi$ has an interior fixed point. This is done after it proves that the essential norm of a composition operator acting on $H^{\infty}$ is either 1 or 0 .
\end{abstract}

\section{Introduction.}

Throughout this paper, $\mathbb{D}$ denotes the unit disk $\{z:|z|<1\}, \varphi$ denotes an analytic self-map of $\mathbb{D}, C_{\varphi}$ is the composition operator defined by $C_{\varphi}(f)=$ $f \circ \varphi,\left\|C_{\varphi}\right\|_{e}$ and $\rho_{e}$ represent the essential norm and essential spectral radius of $C_{\varphi}$ respectively, and $H(\mathbb{D})$ is the space of analytic functions on $\mathbb{D}$.

The essential norm of an operator is the distance from the operator to the space of compact operators. The essential norm of composition operator acting on $H^{2}$, the Hardy space of analytic functions $f$ on $\mathbb{D}$ such that $\int_{-\pi}^{\pi}\left|f\left(e^{i \theta}\right)\right|^{2} d \theta<\infty$, was given by J. H. Shapiro in terms of the Nevanlinna counting function [Sh1]. The spectrum of composition operator on $H^{2}$ has also been studied extensively. Kamowitz was the first to investigate spectrum of composition operator whose symbol is not an inner function and has a fixed point in the disk. He proved in $[\mathbf{K a m}]$ that the spectrum of $C_{\varphi}$ on $H^{2}$ is the set $\left\{\lambda:|\lambda| \leq \rho_{e}\right\} \cup\left\{\varphi^{\prime}(a)^{n}: n \in \mathbb{N}\right\} \cup\{1\}$ if $\varphi$ is analytic in a neighborhood of $\mathbb{D}$, not an inner function and has an interior fixed point $a$. Then Cowen and MacCluer proved in [CM1] the same conclusion in the case that $\varphi$ is univalent, not an automorphism and has an interior fixed point. But a complete understanding of the spectrum of $C_{\varphi}$ on $H^{2}$ is still lacking. While much attention has been devoted to the study of $H^{2}$, the behavior of $C_{\varphi}$ acting on $H^{\infty}$, the space of bounded analytic functions on $\mathbb{D}$, has barely been discussed. It is the purpose of this paper to investigate some properties of $C_{\varphi}$ acting on $H^{\infty}$.

There are two main results of this paper. One of the results, which is stated as Theorem 1, is that the essential norm of $C_{\varphi}$ acting on $H^{\infty}$ is either 1 or 0 . This leads to the corollary that the essential spectral radius of $C_{\varphi}$ on $H^{\infty}$ is also 1 or 0 . Then the two theorems described in the previous paragraph about the spectrum of $C_{\varphi}$ on $H^{2}$, together with this corollary, 
suggest that if $\varphi$ has an interior fixed point $a$, the spectrum of $C_{\varphi}$ on $H^{\infty}$ is $\overline{\mathbb{D}}$ or the sequence $\left\{0,1, \varphi^{\prime}(a)^{n}: n=1,2, \ldots\right\}$. This is the second main result, stated and proved below as Theorem 4. But unlike the theorems about the spectrum of $C_{\varphi}$ acting on $H^{2}$ previously referred to, this does not require $\varphi$ to be univalent or analytic in a neighborhood of $\mathbb{D}$. It only requires $\varphi$ to have an interior fixed point.

\section{Essential norm.}

The essential norm of $C_{\varphi}$ on $H^{\infty}$ is defined to be

$$
\left\|C_{\varphi}\right\|_{e}=\inf \left\{\left\|C_{\varphi}-K\right\|: K \text { is compact operator on } H^{\infty}\right\} .
$$

Clearly $C_{\varphi}$ is compact if and only if its essential norm is zero.

The next result shows that there is only one other possible value for the essential norm of a composition operator on $H^{\infty}$.

Theorem 1. If $C_{\varphi}$ is not compact on $H^{\infty}$, then its essential norm is 1 .

In order to prove this theorem, we need the following lemma, which was first proved by Schwartz [Sch].

Lemma 2. $C_{\varphi}$ is compact on $H^{\infty}$ if and only if $\varphi(\mathbb{D})$ is relatively compact in $\mathbb{D}$.

Proof of Theorem 1. We know that $C_{\varphi}$ is compact if and only if its essential norm is 0 . The main argument here is that the essential norm must be 1 if $C_{\varphi}$ is not compact. Since $\left\|C_{\varphi}(f)\right\|_{\infty}=\sup _{z \in \mathbb{D}}|f \circ \varphi(z)| \leq \sup _{z \in \mathbb{D}}|f(z)|=$ $\|f\|_{\infty},\left\|C_{\varphi}\right\| \leq 1$, and hence $\left\|C_{\varphi}\right\|_{e} \leq 1$. It suffices to prove that $\left\|C_{\varphi}\right\|_{e} \geq 1$ if $C_{\varphi}$ is not compact on $H^{\infty}$.

Now assume $C_{\varphi}$ is not compact on $H^{\infty}$. By Lemma $2, \sup _{z \in \mathbb{D}}|\varphi(z)|=1$. There exists a sequence $\left\{a_{k}\right\}_{k=1}^{\infty} \subset \mathbb{D}$ such that $\varphi\left(a_{k}\right) \rightarrow e^{i \beta}$ as $k \rightarrow \infty$ for some $\beta \in \mathbb{R}$. Without loss of generality, let's assume $e^{i \beta}=1$. Let $\left\{r_{n}\right\}_{n=1}^{\infty}$ be a nonnegative sequence increasing to 1 , and

$$
\psi_{n}(z)=\frac{z-r_{n}}{1-r_{n} z} .
$$

Then $\left\|\psi_{n}\right\|_{\infty}=1, \psi_{n}$ fixes 1 and -1 for all $n \in \mathbb{N}$, and $\psi_{n}(z) \rightarrow-1$ as $n \rightarrow \infty$ for all $z \in \mathbb{D}$. Let $K$ be a compact operator on $H^{\infty}$. We want to show that $\left\|C_{\varphi}-K\right\| \geq 1$. Since $\mathrm{K}$ is compact and $\left\|\psi_{n}\right\|_{\infty}=1$, there is a subsequence $\left\{\psi_{n_{j}}\right\}_{j=1}^{\infty}$ and an $f \in H^{\infty}$ such that $\lim _{j \rightarrow \infty}\left\|K \psi_{n_{j}}-f\right\|_{\infty}=0$. For $\left\|C_{\varphi}-K\right\| \geq 1$ to be true, it is enough to prove that $\lim \sup _{j \rightarrow \infty} \|\left(C_{\varphi}-\right.$ $K)\left(\psi_{n_{j}}\right) \|_{\infty} \geq 1$. But

$$
\left\|\left(C_{\varphi}-K\right)\left(\psi_{n_{j}}\right)\right\|_{\infty} \geq\left\|C_{\varphi}\left(\psi_{n_{j}}\right)-f\right\|_{\infty}-\left\|K \psi_{n_{j}}-f\right\|_{\infty},
$$


which implies

$$
\limsup _{j \rightarrow \infty}\left\|\left(C_{\varphi}-K\right)\left(\psi_{n_{j}}\right)\right\|_{\infty} \geq \limsup _{j \rightarrow \infty}\left\|C_{\varphi}\left(\psi_{n_{j}}\right)-f\right\|_{\infty} .
$$

It suffices to prove that $\lim \sup _{j \rightarrow \infty}\left\|C_{\varphi}\left(\psi_{n_{j}}\right)-f\right\|_{\infty} \geq 1$.

The fact that $\psi_{n}(z) \rightarrow-1$ as $n \rightarrow \infty$ for all $z \in \mathbb{D}$ implies that $\psi_{n_{j}} \circ$ $\varphi(z) \rightarrow-1$ as $j \rightarrow \infty$, and hence $\lim _{j \rightarrow \infty}\left|\psi_{n_{j}} \circ \varphi(z)-f(z)\right|=|-1-f(z)|$ for all $z \in \mathbb{D}$. If there is $z_{0} \in \mathbb{D}$ such that $\left|-1-f\left(z_{0}\right)\right| \geq 1$, we have $\left\|\psi_{n_{j}} \circ \varphi-f\right\|_{\infty} \geq\left|\psi_{n_{j}} \circ \varphi\left(z_{0}\right)-f\left(z_{0}\right)\right| \rightarrow\left|-1-f\left(z_{0}\right)\right| \geq 1$, which implies $\lim \sup _{j \rightarrow \infty}\left\|\psi_{n_{j}} \circ \varphi-f\right\|_{\infty} \geq 1$ as desired. Otherwise, $|-1-f(z)|<1$ for all $z \in \mathbb{D}$. Then by the triangle inequality $|1-f(z)|>1$ for all $z \in \mathbb{D}$. Consider the sequence $\left\{a_{k}\right\}_{k=1}^{\infty} \subset \mathbb{D}$ which was obtained at the beginning of the proof. We have $\lim _{k \rightarrow \infty} \varphi\left(a_{k}\right)=1$ and $\left\{f\left(a_{k}\right)\right\}_{k=1}^{\infty}$ is bounded since $f \in H^{\infty}$. Then there is a subsequence $\left\{f\left(a_{k_{j}}\right)\right\}_{j=1}^{\infty}$ converging to some $\omega \in \mathbb{C}$. By reindexing we may assume, without loss of generality that, $\lim _{k \rightarrow \infty} f\left(a_{k}\right)=\omega$. Then by our assumption on $f,|1-\omega|=\lim _{k \rightarrow \infty}\left|1-f\left(a_{k}\right)\right| \geq 1$. Since $\psi_{n}$ is continuous, $\psi_{n}(1)=1$ and $\lim _{k \rightarrow \infty} \varphi\left(a_{k}\right)=1$, it follows that $\lim _{k \rightarrow \infty} \mid \psi_{n}$ 。 $\varphi\left(a_{k}\right)-f\left(a_{k}\right)|=| 1-\omega \mid \geq 1$ for all $n$. Then $\left\|\psi_{n_{j}} \circ \varphi-f\right\|_{\infty} \geq \lim _{k \rightarrow \infty} \mid \psi_{n_{j}} \circ$ $\varphi\left(a_{k}\right)-f\left(a_{k}\right) \mid \geq 1$ for all $j$. Hence $\lim _{\sup _{j \rightarrow \infty}}\left\|\psi_{n_{j}} \circ \varphi-f\right\|_{\infty} \geq 1$ as desired.

If $e^{i \beta} \neq 1$, let $\Psi_{n}(z)=e^{i \beta} \psi_{n}\left(e^{-i \beta} z\right)$. The same proof holds with $\psi_{n}$ replaced by $\Psi_{n}$, and the boundary points 1 and -1 replaced by $e^{i \beta}$ and $-e^{i \beta}$ respectively. This completes the proof of Theorem 1.

For the rest of this paper, $\varphi_{n}$ will denote the $n^{\text {th }}$ iterate of $\varphi$, i.e., $\varphi_{1}=\varphi$ and $\varphi_{n}=\varphi \circ \varphi_{n-1}$ for $n>1$.

Definition ([CM2, p. 150]). If $T$ is a bounded linear operator on a Hilbert space, then the spectrum of the equivalence class in the Calkin algebra that contains $T$ is called the essential spectrum of $T$.

Corollary 3. The essential spectral radius of $C_{\varphi}$ on $H^{\infty}$ is either 1 or 0 . If $C_{\varphi_{n}}\left(=C_{\varphi}^{n}\right)$ is compact for some $n \geq 1$, then $\rho_{e}\left(C_{\varphi}\right)=0$. Otherwise $\rho_{e}\left(C_{\varphi}\right)=1$.

Proof. The conclusion follows immediately from Theorem 1 and the formula that $\rho_{e}\left(C_{\varphi}\right)=\lim _{n \rightarrow \infty}\left(\left\|C_{\varphi}^{n}\right\|_{e}\right)^{1 / n}=\lim _{n \rightarrow \infty}\left(\left\|C_{\varphi_{n}}\right\|_{e}\right)^{1 / n}$.

\section{Spectrum.}

For $C_{\varphi}$ acting on $H^{\infty}$, the spectrum $\sigma\left(C_{\varphi}\right)$ is contained in $\overline{\mathbb{D}}$. This is because the norm of $C_{\varphi}$ acting on $H^{\infty}$ is always 1 , which implies the spectral radius $\rho\left(C_{\varphi}\right)=\lim _{n \rightarrow \infty}\left\|C_{\varphi_{n}}\right\|^{1 / n}=1$.

Theorem 4. If $\varphi$ is not a constant, not an automorphism, and $\varphi(a)=a$ for some $a \in \mathbb{D}$, then

$$
\sigma\left(C_{\varphi}\right)=\overline{\mathbb{D}}, \text { if }\left\|\varphi_{n}\right\|_{\infty}=1 \text { for all } n \in \mathbb{N}
$$


and

$$
\sigma\left(C_{\varphi}\right)=\left\{\varphi^{\prime}(a)^{k}: k=1,2, \ldots\right\} \cup\{0,1\} \text {, if }\left\|\varphi_{n}\right\|_{\infty}<1 \text { for some } n \in \mathbb{N} \text {. }
$$

The proof of Theorem 4 will be given after some lemmas. Some ideas and approaches used in the proof are suggested by the work of Kamowitz in [Kam], and that of Cowen and MacCluer in [CM1].

The following lemma, Lemma 5, follows immediately from Koenigs' Theorem [Koe] (see also [Sh2, Chapter 6]), if we can show that the Koenigs' function $\xi$ of $\varphi$ is in $H^{\infty}$ under the assumption of the Lemma. Since $\xi \circ \varphi=\varphi^{\prime}(a) \xi$, which implies $\xi \circ \varphi_{n}=\varphi^{\prime}(a)^{n} \xi$, and hence $\xi=\varphi^{\prime}(a)^{-n} \xi\left(\varphi_{n}\right)$ for all $n \in \mathbb{N}$, we conclude that $\xi$ is in $H^{\infty}$ under the assumption that $\left\|\varphi_{n}\right\|_{\infty}<1$ for some $n \in \mathbb{N}$.

Lemma 5. For $\varphi$ as in Theorem 4 , suppose $\left\|\varphi_{n}\right\|_{\infty}<1$ for some $n \in \mathbb{N}$. If $\varphi^{\prime}(a) \neq 0$, then $\left\{\varphi^{\prime}(a)^{n}, n=0,1,2, \ldots\right\}$ is the set of eigenvalues of $C_{\varphi}$ on $H^{\infty}$. If $\varphi^{\prime}(a)=0$, the only eigenvalue of $C_{\varphi}$ is 1 .

Lemma 6. Let $\varphi$ be the same as in Theorem 4 and suppose $\left\|\varphi_{n}\right\|_{\infty}<1$ for some $n \in \mathbb{N}$. Then for $C_{\varphi}$ on $H^{\infty}$,

$$
\sigma\left(C_{\varphi}\right)=\left\{\varphi^{\prime}(a)^{k}: k=1,2, \ldots\right\} \cup\{0,1\} .
$$

Proof. Under the hypothesis that $\left\|\varphi_{n}\right\|_{\infty}<1, C_{\varphi_{n}}=C_{\varphi}^{n}$ is compact, which implies that $C_{\varphi}$ is not invertible and hence 0 is in the spectrum. Also, since $C_{\varphi}^{n}$ is compact, $C_{\varphi}$ is a Riesz operator. So its nonzero spectrum consists of eigenvalues [Kön, p. 19-21], and the result follows from Lemma 5.

Lemma 7. Suppose $\varphi(0)=0$. Then $H_{m}=z^{m} H^{\infty}$ is an invariant subspace of $C_{\varphi}$ and $\sigma\left(C_{m}\right) \subset \sigma\left(C_{\varphi}\right)$ where $C_{m}=\left.C_{\varphi}\right|_{H_{m}}$.

Proof. It's easy to see that $H_{m}$ is invariant under $C_{\varphi}$. Since $\varphi(0)=0$, $\varphi(z)=z \phi(z)$ for some $\phi \in H^{\infty}$. Then if $f \in H^{\infty}, C_{\varphi}\left(z^{m} f\right)=\varphi^{m}(f \circ \varphi) \in$ $H_{m}$.

Suppose $\lambda$ is in the spectrum of $C_{m}$. If $\lambda$ is an eigenvalue of $C_{m}$, it must be an eigenvalue of $C_{\varphi}$ and hence in the spectrum of $C_{\varphi}$. If $\lambda$ is not an eigenvalue of $C_{m}$, then $C_{m}-\lambda I$ is one-one. But it is not invertible, and hence not onto. So there exists $f \in H_{m}$ with $f \notin\left(C_{m}-\lambda I\right)\left(H_{m}\right)$. If we can show that $C_{\varphi}-\lambda I$ on $H^{\infty}$ is not onto, then it will follow that $\lambda \in \sigma\left(C_{\varphi}\right)$ and the conclusion holds. Suppose to the contrary $C_{\varphi}-\lambda I$ is onto. Then $f \in\left(C_{\varphi}-\lambda I\right)\left(H^{\infty}\right)$ and there is $g \in H^{\infty}$ with $g \notin H_{m}$ such that $\left(C_{\varphi}-\lambda I\right) g=f$. Let $g=g_{1}+g_{2}$, where $g_{1} \in \operatorname{span}\left(1, z, z^{2}, \ldots, z^{m-1}\right)$ and $g_{2} \in H_{m}$. We have $g_{1} \neq 0$ since $g \notin H_{m}$. Let $f_{1}=\left(C_{\varphi}-\lambda I\right) g_{1}=f-\left(C_{\varphi}-\lambda I\right) g_{2}$. Then $f_{1} \in H_{m}$ since $f, g_{2} \in H_{m}$.

Also by the assumption that $C_{\varphi}-\lambda I$ is onto, for each function $z^{i}, i=$ $1,2, \ldots, m-1$, there exists $h_{i} \in H^{\infty}$ such that $\left(C_{\varphi}-\lambda I\right) h_{i}(z)=z^{i}$. Let $h_{i}=k_{i}+l_{i}$ where $k_{i} \in \operatorname{span}\left(1, z, z^{2}, \ldots, z^{m-1}\right)$ and $l_{i} \in H_{m}$. The next 
step is to show that $g_{1}, k_{0}, k_{1}, \ldots, k_{m-1}$ are linearly independent. Suppose $\beta g_{1}+\sum_{i=0}^{m-1} \alpha_{i} k_{i}=0$ for some $\beta$ and $\alpha_{i}, i=0,1, \ldots, m-1$. Then $\beta g_{1}+$ $\sum_{i=0}^{m-1} \alpha_{i} h_{i}=\left(\beta g_{1}+\sum_{i=0}^{m-1} \alpha_{i} k_{i}\right)+\sum_{i=0}^{m-1} \alpha_{i} l_{i}=\sum_{i=0}^{m-1} \alpha_{i} l_{i} \in H_{m}$. So $\left(C_{\varphi}-\lambda I\right)\left(\beta g_{1}(z)+\sum_{i=0}^{m-1} \alpha_{i} h_{i}(z)\right)=\beta f_{1}(z)+\sum_{i=0}^{m-1} \alpha_{i} z^{i} \in H_{m}$. Since $f_{1} \in H_{m}$, we have $\alpha_{i}=0$ for $i=0,1, \ldots, m-1$. Then it follows that $\beta=0$. So $g_{1}, k_{0}, k_{1}, \ldots, k_{m-1}$ are linearly independent. But this is impossible since $\left\{g_{1}, k_{0}, k_{1}, \ldots, k_{m-1}\right\} \subset \operatorname{span}\left(1, z, \ldots, z^{m-1}\right)$, which is only $m$ dimensional. So $C_{\varphi}-\lambda I$ is not onto, and hence $\lambda$ is in the spectrum of $C_{\varphi}$.

Definition. We say the sequence of points $\left\{z_{k}\right\}_{k=K}^{M}$ is an iteration sequence for $\varphi$ if $\varphi\left(z_{k}\right)=z_{k+1}$ for $K \leq k<M$ where $K \geq-\infty$ and $M \leq \infty$.

Lemma 8 ([CM2, p. 292, Lemma 7.34]). If $\varphi$ is not an automorphism and $\varphi(0)=0$, then given $0<r<1$, there exists $M_{r}$ with $1 \leq M_{r}<\infty$ such that if $\left\{z_{k}\right\}_{-K}^{\infty}$ is an iteration sequence with $\left|z_{l}\right| \geq r$ for some $l \geq 0$ and if $\left\{w_{k}\right\}_{-K}^{l}$ is arbitrary, there is $h \in H^{\infty}$ such that $h\left(z_{k}\right)=w_{k}$ for $-K \leq k \leq l$ and $\|h\|_{\infty} \leq M_{r} \sup \left\{\left|w_{k}\right|:-K \leq k \leq l\right\}$.

Lemma 9 ([CM2, p. 293, Lemma 7.35]). For $\varphi$ in Lemma 8 and $\left\{z_{k}\right\}$ any iteration sequence, there exists $c<1$ such that $\left|z_{k+1}\right| /\left|z_{k}\right| \leq c$ whenever $\left|z_{k}\right| \leq 0.5$.

Proof of Theorem 4. The statement in the case that $\left\|\varphi_{n}\right\|_{\infty}<1$ for some $n \in \mathbb{N}$ is the result of Lemma 6 . Now suppose $\left\|\varphi_{n}\right\|_{\infty}=1$ for all $n \in$ $\mathbb{N}$. We want to prove $\sigma\left(C_{\varphi}\right)=\overline{\mathbb{D}}$. If the interior fixed point $a \neq 0$, let $\tau(z)=(a-z) /(1-\bar{a} z)$ and $\psi=\tau \circ \varphi \circ \tau$. Then $\tau^{-1}=\tau, \psi(0)=0$ and $C_{\psi}=C_{\tau} \circ C_{\varphi} \circ C_{\tau}=C_{\tau} \circ C_{\varphi} \circ C_{\tau}^{-1} . C_{\varphi}$ and $C_{\psi}$ are similar and hence have the same spectrum. So without loss of generality, we can assume $\varphi(0)=0$.

Since $\sigma\left(C_{\varphi}\right) \subset \overline{\mathbb{D}}$ and $\sigma\left(C_{\varphi}\right)$ is closed, it suffices to prove $\mathbb{D}-\{0\} \subset \sigma\left(C_{\varphi}\right)$. Let $0 \neq \lambda \in \mathbb{D}, H_{m}=z^{m} H^{\infty}$, and $C_{m}=\left.C_{\varphi}\right|_{H_{m}}$. By Lemma 7 , it suffices to prove that $\lambda$ is in the spectrum of $C_{m}$ for some positive integer $m$. Since $C_{m}-\lambda I$ is not onto if and only if $\left(C_{m}-\lambda I\right)^{*}$ is not bounded from below, it is enough to find a positive integer $m$ with $\left(C_{m}-\lambda I\right)^{*}$ not bounded from below.

Let $M$ be the constant $M_{r}$ in Lemma 8 corresponding to $\mathrm{r}=0.25$ and suppose we have an iteration sequence $\left\{z_{k}\right\}_{-K}^{\infty}$ with $K \geq 0$ and $\left|z_{0}\right|>0.5$ (this sequence will be determined later on). Let $n=\max \left\{k:\left|z_{k}\right| \geq 0.25\right\}$. Then $n \geq 0$ and $\left|z_{k}\right|<0.25<0.5$ for all $k \geq n+1$. By Lemma 9 , there is a number $c_{1}<1$ so that $\left|z_{k+1}\right| \leq c_{1}\left|z_{k}\right|$ whenever $k \geq n+1$. If $z_{n} \leq 0.5$, the inequality also holds for $k=n$. If $\left|z_{n}\right|>0.5$, since $\left|z_{n+1}\right|<0.25$, we have $\left|z_{n+1}\right|<0.5\left|z_{n}\right|$. Let $c=\max \left\{c_{1}, 0.5\right\}$. Then $\left|z_{k+1}\right| \leq c\left|z_{k}\right|$ for all $k \geq n$. It follows that $\left|z_{k}\right| \leq c^{k-n}\left|z_{n}\right|$ whenever $k \geq n$. Since $c<1$, there exists a positive integer $m$ such that $c^{m} /|\lambda|<1 /(2 M+1)<1$. For this $m,\left(C_{m}-\lambda I\right)^{*}$ is not bounded from below. To see that, let $\varepsilon>0$ and we will construct a bounded linear functional $L_{\lambda}$ on $H_{m}$ with $\left\|\left(C_{m}-\lambda I\right)^{*} L_{\lambda}\right\| /\left\|L_{\lambda}\right\|<\varepsilon$. 
Let's define $L_{\lambda}$ by $L_{\lambda}(f)=\sum_{k=-K}^{\infty} \lambda^{-k} f\left(z_{k}\right)$ for $f \in H_{m}$. We will see that $L_{\lambda}$ is well-defined and indeed it is bounded.

For $f \in H_{m}, z^{-m} f(z)$ is analytic and $\|f\|_{\infty}=\left\|z^{-m} f(z)\right\|_{\infty}$. So

$$
\left|f\left(z_{k}\right)\right|=\left|z_{k}\right|^{m}\left|z_{k}^{-m} f\left(z_{k}\right)\right| \leq\left|z_{k}\right|^{m}\left\|z^{-m} f(z)\right\|_{\infty}=\left|z_{k}\right|^{m}\|f\|_{\infty} .
$$

Then

$$
\begin{aligned}
\sum_{k=-K}^{\infty}|\lambda|^{-k}\left|f\left(z_{k}\right)\right| & \leq\|f\|_{\infty} \sum_{k=-K}^{\infty}|\lambda|^{-k}\left|z_{k}\right|^{m} \\
& =\|f\|_{\infty}\left(\sum_{k=-K}^{n}|\lambda|^{-k}\left|z_{k}\right|^{m}+\sum_{k=n+1}^{\infty}|\lambda|^{-k}\left|z_{k}\right|^{m}\right) .
\end{aligned}
$$

For $k>n,\left|z_{k}\right| \leq c^{k-n}\left|z_{n}\right|$ and so $\left|z_{k}\right|^{m} \leq\left(c^{m}\right)^{k-n}\left|z_{n}\right|^{m}$. We see that

$$
\sum_{k=n+1}^{\infty}|\lambda|^{-k}\left|z_{k}\right|^{m} \leq \sum_{k=n+1}^{\infty} \frac{\left(c^{m}\right)^{k-n}\left|z_{n}\right|^{m}}{|\lambda|^{k-n}|\lambda|^{n}}=\frac{\left|z_{n}\right|^{m}}{|\lambda|^{n}} \sum_{k=n+1}^{\infty}\left(\frac{c^{m}}{|\lambda|}\right)^{k-n}<\infty .
$$

It follows that $\sum_{k=-K}^{\infty} \lambda^{-k} f\left(z_{k}\right)$ converges. Hence $L_{\lambda}$ is well-defined and by (1) it is bounded.

Now let's estimate $\frac{\left\|\left(C_{m}-\lambda I\right)^{*} L_{\lambda}\right\|}{\left\|L_{\lambda}\right\|}$. For $f \in H_{m}$,

$$
\begin{aligned}
\left\langle f,\left(C_{m}-\lambda I\right)^{*} L_{\lambda}\right\rangle & =\left\langle\left(C_{m}-\lambda I\right) f, L_{\lambda}\right\rangle \\
& =\left\langle f \circ \varphi-\lambda f, L_{\lambda}\right\rangle \\
& =\sum_{k=-K}^{\infty} \lambda^{-k}\left(f \circ \varphi\left(z_{k}\right)-\lambda f\left(z_{k}\right)\right) \\
& =\sum_{k=-K}^{\infty}\left(\lambda^{-k} f\left(z_{k+1}\right)-\lambda^{-(k-1)} f\left(z_{k}\right)\right) \\
& =-\lambda^{K+1} f\left(z_{-K}\right) .
\end{aligned}
$$

Then

$$
\begin{aligned}
\left\|\left(C_{m}-\lambda I\right)^{*} L_{\lambda}\right\| & =\sup _{0 \neq f \in H_{m}} \frac{\left|\left\langle f,\left(C_{m}-\lambda I\right)^{*} L_{\lambda}\right\rangle\right|}{\|f\|_{\infty}} \\
& =\sup _{\substack{0 \neq f \in H_{m}\\
}} \frac{|\lambda|^{K+1}\left|f\left(z_{-K}\right)\right|}{\|f\|_{\infty}} \\
& \leq|\lambda|^{K+1} .
\end{aligned}
$$

We also need a lower bound for $\left\|L_{\lambda}\right\|$. If we apply Lemma 8 to the iteration sequence $\left\{z_{k}\right\}_{-K}^{\infty}$ with $\mathrm{r}=0.25$, we can find a function $h \in H^{\infty}$ with $\|h\|_{\infty} \leq$ $M,\left|h\left(z_{k}\right)\right|=1$ and $\lambda^{-k} z_{k}^{m} h\left(z_{k}\right)>0$ for $-K \leq k \leq n$. Let $g(z)=z^{m} h(z) \in$ 
$H_{m}$. Then $\|g\|_{\infty} \leq M$ and

$$
\begin{aligned}
L_{\lambda}(g) & =\sum_{k=-K}^{\infty} \lambda^{-k} z_{k}^{m} h\left(z_{k}\right) \\
& =\sum_{k=-K}^{n-1}|\lambda|^{-k}\left|z_{k}\right|^{m}+|\lambda|^{-n}\left|z_{n}\right|^{m}+\sum_{k=n+1}^{\infty} \lambda^{-k} z_{k}^{m} h\left(z_{k}\right) .
\end{aligned}
$$

By the estimate in (2) and because $M \geq 1$ and $c^{m} /|\lambda|<1 /(2 M+1)$ from the choice of $m$, we have

$$
\begin{aligned}
\left|\sum_{k=n+1}^{\infty} \lambda^{-k} z_{k}^{m} h\left(z_{k}\right)\right| & \leq \sum_{k=n+1}^{\infty}|\lambda|^{-k}\left|z_{k}\right|^{m}\left|h\left(z_{k}\right)\right| \\
& \leq M|\lambda|^{-n}\left|z_{n}\right|^{m} \sum_{k=n+1}^{\infty}\left(\frac{c^{m}}{|\lambda|}\right)^{k-n} \\
& =M|\lambda|^{-n}\left|z_{n}\right|^{m} \frac{\frac{c^{m}}{|\lambda|}}{1-\frac{c^{m}}{|\lambda|}} \\
& \leq M|\lambda|^{-n}\left|z_{n}\right|^{m} \frac{\frac{1}{2 M+1}}{1-\frac{1}{2 M+1}} \\
& =\frac{1}{2}|\lambda|^{-n}\left|z_{n}\right|^{m} .
\end{aligned}
$$

This shows that

$$
\begin{aligned}
\left|L_{\lambda}(g)\right| & \geq \sum_{k=-K}^{n-1}|\lambda|^{-k}\left|z_{k}\right|^{m}+|\lambda|^{-n}\left|z_{n}\right|^{m}-\left|\sum_{k=n+1}^{\infty} \lambda^{-k} z_{k}^{m} h\left(z_{k}\right)\right| \\
& \geq \sum_{k=-K}^{n-1}|\lambda|^{-k}\left|z_{k}\right|^{m}+\frac{1}{2}|\lambda|^{-n}\left|z_{n}\right|^{m} \geq \frac{1}{2}\left|z_{0}\right|^{m} .
\end{aligned}
$$

Then

$$
\left\|L_{\lambda}\right\| \geq \frac{\left|L_{\lambda}(g)\right|}{\|g\|_{\infty}} \geq \frac{\left|z_{0}\right|^{m}}{2 M} \geq \frac{(0.5)^{m}}{2 M} .
$$

It follows that

$$
\frac{\left\|\left(C_{m}-\lambda I\right)^{*} L_{\lambda}\right\|}{\left\|L_{\lambda}\right\|} \leq \frac{2 M|\lambda|^{K+1}}{0.5^{m}} .
$$

Since $|\lambda|<1$, this is less than $\varepsilon$ if we choose $K$ sufficiently large. For the chosen $K$, we can determine the iteration sequence $\left\{z_{k}\right\}_{-K}^{\infty}$. Since $\left\|\varphi_{K}\right\|_{\infty}=$ 1 by assumption, there exists $w \in \mathbb{D}$ with $\left|\varphi_{K}(w)\right|>0.5$. Let $z_{-K}=w$ and $z_{k+1}=\varphi\left(z_{k}\right)$ for $k>-K$. Then $\left|z_{0}\right|=\left|\varphi_{K}\left(z_{-K}\right)\right|>0.5$. The above calculation follows, thus $\left(C_{m}-\lambda I\right)^{*}$ is not bounded from below as desired. 
Acknowledgement. I would like to thank my advisor Professor Wayne Smith for his valuable suggestions and patient guidance in my writing this paper.

\section{References}

[Con] J.B. Conway, A Course in Functional Analysis, second edition, Springer-Verlag, 1990, MR 91e:46001, Zbl 0706.46003.

[CM1] C. Cowen and B. MacCluer, Spectra of some composition operators, Journal of Functional Analysis, 125 (1994), 223-251, MR 95i:47058, Zbl 0814.47040.

[CM2] _ Composition Operator on Spaces of Analytic Functions, CRC Press, 1995, MR 97i:47056, Zbl 0873.47017.

[Kam] H. Kamowitz, The spectra of composition operators on $H^{p}$, Journal of Functional Analysis, 18 (1975), 132-150, MR 53 \#11417, Zbl 0295.47003.

[Koe] G. Koenig, Recherches sur les intégrales de certaines équations functionelles, Ann. Sci École Norm. Sup. (Sér. 3), 1 (1884), supplément, 3-41.

[Kön] H. König, Eigenvalue Distribution of Compact Operators, Birkhauser Verlag, 1986, MR 88j:47021, Zbl 0618.47013

[Sch] H.J. Schwartz, Composition operator on $H^{p}$, Thesis, University of Toledo, 1969.

[Sh1] J.H. Shapiro, The essential norm of a composition operator, Annals of Mathematics, 125 (1987), 375-404, MR 88c:47058, Zbl 0642.47027.

[Sh2] , Composition Operators and Classical Function Theory, Springer-Verlag, 1993, MR 94k:47049, Zbl 0791.30033.

Received March 1, 2000 and revised October 2, 2000.

1111 Balclutha Dr, \# J204

Foster City, CA 94404

E-mail address: zheng_lixin@hotmail.com 